\title{
Computational identification of miRNAs and their targets from Niger (Guizotia abyssinica)
}

\author{
K. Y. Prathiba ${ }^{1}$, S. Usha ${ }^{2,3}$, B. Suchithra ${ }^{2,4}$, M. N. Jyothi ${ }^{2,3}$, V. R. Devaraj ${ }^{4}$, R. Nageshbabu ${ }^{2 *}$ \\ ${ }^{1}$ Department of Botany, Maharani's Science College for Women, Bangalore-560001, India. ${ }^{2}$ Post Graduate Department of Biochemistry, Maharani's \\ Science College for Women, Bangalore-560001, India. ${ }^{3}$ School of Biological Engineering and Sciences, Shobhit University, Meerut, India. ${ }^{4}$ Department of \\ Biochemistry, Central College Campus, Bangalore University, Bangalore -560001 India.
}

\begin{tabular}{|c|c|}
\hline ARTICLE INFO & ABSTRACT \\
\hline $\begin{array}{l}\text { Article history: } \\
\text { Received on: } 21 / 10 / 2016 \\
\text { Accepted on: } 02 / 12 / 2016 \\
\text { Available online: } 20 / 03 / 2017\end{array}$ & $\begin{array}{l}\text { MicroRNAs play a pivotal role in regulating a broad range of biological processes, acting by cleaving mRNAs or } \\
\text { by translational repression. A group of plant microRNAs are evolutionarily conserved; however, others are } \\
\text { expressed in a species-specific manner. In this study we used homology-based analysis with available expressed } \\
\text { sequence tag (EST) of Niger (Guizotia abyssinica) to predict conserved miRNAs. Two potent miRNAs targeting }\end{array}$ \\
\hline $\begin{array}{l}\text { Key words: } \\
\text { EST; GO analysis; miRNA; } \\
\text { Transcription factors. }\end{array}$ & $\begin{array}{l}49 \text { genes were identified. The newly identified miRNAs belongs to miR } 2592 \text { and miR396 family. Targets } \\
\text { recognized were F-box proteins, leucine zipper, DEAD box RNA helicase, disease resistant proteins. Gene } \\
\text { annotations revealed miRNAs were involved in growth and development and Encyclopaedia of Genes and } \\
\text { Genomes (KEGG) pathway analyses showed miRNAs were involved in metabolic pathways. }\end{array}$ \\
\hline
\end{tabular}

\section{INTRODUCTION}

miRNAs are small endogenous 20-24nt non-coding RNAs derived from single-stranded RNA precursors that can form stemloop structures and have been proved to play a vital role in wide range of biological process. In plants, after transcription by Pol II or Pol III enzyme into primary miRNA (pri-miRNA), the miRNA gene is processed by Dicer-like (DCL) into a stem-loop that contain miRNA/ miRNA* duplex, called an miRNA precursor (pre-miR-NA). Subsequently, the duplex is cleaved from the pre-miRNA and transported from the nucleus into the cytoplasm which combines with Argonaute (AGO) forming the RNA-induced silencing complex (RISC). miRNAs, regulate the gene expression at post-transcriptional level by directing the mRNA cleavage or by repressing translation [1,2]. In plants, miRNAs regulate their target mRNAs by nearly perfect complementary base pairing, which leads to the degradation of target genes [3]. Several biological experiments indicate that

\footnotetext{
* Corresponding Author

Nagesh babu R, Assistant Professor Postgraduate Department of Biochemistry Maharani's Science College for Women, Palace Road, Bangalore-560001 India. Email: nageshbabur@gmail.com
}

miRNAs play key roles during development and in response to environmental stresses [4, 5]. There are different methods in identifying miRNAs; viz. direct cloning, high throughput sequencing and computational analysis which uses ESTs and GSS sequences. Although high throughput sequencing technology has made miRNA identification rapid and significant, computational analysis is also a promising way in identification of conserved and novel miRNAs. A majority of miRNAs are evolutionary conserved, which can be identified by sequence homology analyses [6]. Nevertheless, a proportion of miRNAs are species-specific and usually expressed at lower levels in comparison with other conserved miRNAs [7, 8]. Till date, miRNAs have been identified in variety of species using computational approach such as silkworm, where 16 novel miRNAs were identified using homology search of Genomic survey sequence (GSS) [9], 8 potential novel miRNAs were identified in Festuca arundinacea using ESTs and GSS [10], 6 miRNAs that regulate twenty potential targets were predicted in mulberry [11], in Soybean 521 novel miRNA genes belonging to 58 families were identified [12]. Three conserved miRNAs belonging to miR166 and miR1310 were identified in Finger millet using ESTs [13]. Niger (Guizotia abyssinica (L. f.) Cass, Compositae) is a dicotyledonous herb and oilseed crop cultivated in Ethiopia and India. 
Niger is usually grown on light poor soils with coarse texture. Unlike other plants Niger also faces abiotic and biotic stress in changing environmental conditions; hence studies on miRNAs in this plant, an untouched area till date will help in improving the crop yield and quality.

In the present study, for first time we have identified two conserved miRNAs belonging to miR396 and miR2592 family using available ESTs and characterized their target genes. Gene ontology, protein interactions and KEGG pathway analysis for target genes were also performed with available computational tools.

\section{METHODOLOGY}

\subsection{Identification of conserved miRNA}

EST sequences of Niger (Guizotia abyssinica) were downloaded from NCBI (http://www.ncbi.nlm.nih.gov) and plant miRNAs from miRBase v21 (www.mirbase.org) were used as reference for predicting the conserved miRNAs. The sequences were assembled using CAP3 program [14]. tRNA and rRNA sequences were filtered with successive BLAST searches over the ribosomal RNAs database from 12 (http://www.sanger.ac.uk/Software/Rfam/). The coding regions were removed using BLASTX. mirnaDetect (http://datamining.xmu.edu.cn/main/ leyiwei/mirnaDetect.html) a tool for detecting potential pre-miRNAs from the genome-scale data was also used to predict the possible miRNAs in Niger [15]. The secondary structures of putative pre-miRNAs were predicted by Mfold (http://mfold.rna.albany.edu) [16].

\subsection{Target Prediction and GO analysis}

Target predictions for the miRNAs are based on the principle of nearly perfect complementation between the miRNA and target mRNAs. The Brassica rapa transcripts, downloaded from phytozome v10 (www.phytozome.net) was used to determine the potential target mRNA candidates for miRNAs using psRNATarget with default parameters (http://www.plantgrn.org/psRNATarget/) [17]. Sequences with a score of less than 4 were regarded as miRNA target genes. Functional annotations of predicted targets were analysed using BGI WEGO platform (http://wego.genomics.org.cn/cgibin/wego/index.pl) [18].

\subsection{KEGG pathway analysis and predicted protein interactions}

The KEGG (Kyoto Encyclopedia of Genes and Genomes) database was used to identify the significantly enriched pathways of miRNA target genes. STRING (http://string-db.org/) computational tool was used to study protein-protein interactions for miRNA targets. These interactions are derived from genomic context, high throughput experiments and Co-expressions.

\section{RESULTS AND DISCUSSION}

\subsection{Identification of conserved miRNAs in Niger}

Till date there are 25711 Niger ESTs available in NCBI, which were downloaded for the analysis. microRNAs in Niger were identified using mirnaDetect and CAP3 tools. Initially all the ESTs sequences were assembled using CAP3 programme and Blast search was initiated with default parameters. Initially we found more than 32 probable miRNA candidates which were screened for their precursors, which were supposed to be the parent molecules of functional miRNAs. The obtained sequences were filtered for the tRNA, ribosomal RNA contamination by successive Blastn searches on Sanger's Rfam database. The presence of coding sequences among the predicted originator sequences were analysed through Blastx strategy against nonredundant protein database. Successive filtering resulted in 13 microRNAs, which were screened for stable secondary stem-loop structures, as the main feature of miRNA is to fold back with precursor sequences. Stem-loops were obtained from Zuker folding algorithm adopted form MFOLD 3.2. Out of 13 putative pre-miRNAs only 02 sequences satisfied the criteria described by Ambros et al. [19], which are 21nt in length, which is consistent with other reports [20-21]. The identified conserved miRNAs in Niger were designated as Gab-miR1 and Gab-miR2 which belonged to the family miR2592 and miR396 (Table 1). Precursor sequence of identified mature Gab-miR1 and Gab-miR2 was 165 and 120nt in length with the MEF of -41.1 and $-26.5 \mathrm{kcal} / \mathrm{mol}$ respectively. Precursor sequences for identified miRNAs are given in Supl. 1 and their secondary structures are depicted in Figure 1.

Table 1 Conserved miRNAs identified in Niger by EST analysis.

\begin{tabular}{ccccccc}
\hline miRNA & Gene ID & Sequence & Length & Precursor length & GC\% & MFE* \\
\hline Gab-miR1 & gb|GE573440.1 & AAAUGCUUGAGUCCUGUUGUU & 21 & 165 & 40 & -41.1 \\
Gab-miR2 & $\mathrm{gb} \mid$ GE552120.1 & UUCCACAGCUUUCUUGAACUU & 21 & 120 & 34 & -26.5 \\
\hline
\end{tabular}

Suppl. 1: Precursor sequences of conserved miRNAs identified in Niger.

\begin{tabular}{cl}
\hline miRNA & \multicolumn{1}{c}{ Precursor Sequence } \\
\hline Gab-miR1 & GGAAGAGUAUUAGGGCUUGCUAGAGAUAAGGAUCUUGGAGUGGAGAACGUGAGGCAUUUUAUUCUCGAUGAGUGUGACA \\
& AAAUGCUUGAGUCACUUGAUAUGAGAAGAGAUGUUCAGGAGAUUUUUAAGAUGACACCUCAUGACAAGCAAGUAAUGAU \\
& GUUCUCU \\
\hline Gab-miR2 & AUUGAUAUCAGAUGAGAGCUUCAUCUUCUUCUUCAUCUUCUGAUGAUGAAUUUCUUCAAGAAACUGAGAAUUGAACAACA \\
& CCCAGAUCAAGAAACAAGAUCAGAAGCUCAAAUCUUCAAU \\
\hline
\end{tabular}



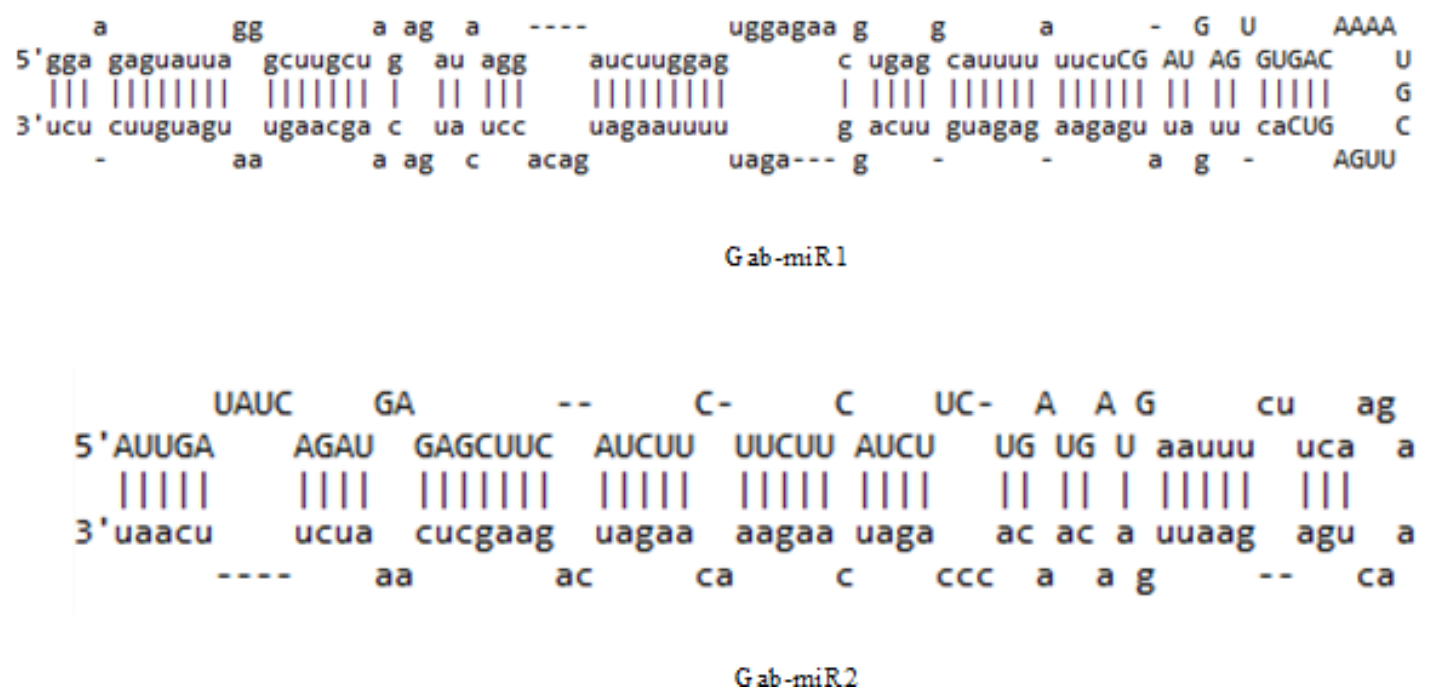

Fig. 1: Secondary structure of predicted conserved miRNAs in Niger.

\subsection{Target prediction and GO analysis}

Functional importance of the predicted miRNAs can be understood by studying their targets, targets identified using insilco approaches have been important till date. The systemic search for mRNA complementary to miRNAs led to the observation that many miRNAs targeted genes through perfect/nearly perfect matches in anti-sense manner leading to degradation of the target gene [22]. psRNATarget scan was used to identify targets in Niger with Brassica rapa transcripts. A total of 49 targets were identified for two conserved miRNAs, and the main mechanism of gene regulation by miRNA was through target degradation as evidenced by $92 \%$ of target cleavage. Both GabmiR1 and Gab-miR2 showed multiple targets, Gab-miR1 targeted HMG box DNA binding protein, F-box protein and cytochrome p450. Gab-miR2 targeted DEAD box RNA helicase, carboxyltransferace, disease resistant protein, growth regulating factors, heat shock proteins etc. (Suppl. 2). In Arabidopsis HMG domain containing SSRP1 was found to be required in DNA demthylation and for activation and repression of many parentally imprinted genes in the central cell [23]. Recently in Arabidopsis it was shown that AT-rich interaction domain and the HMG-box domain of ARID-HMG proteins promote DNA mini-circle formation but are also capable of inducing negative supercoils into relaxed plasmid DNA suggesting the involvement of this protein in several nuclear events [24]. F-box proteins regulate diverse cellular processes, including cell cycle transition, transcriptional regulation and signal transduction, by playing roles in Skp1pcullin-F-box protein (SCF) complexes or non-SCF complexes. Stefanowicz et al., showed that in Arabidopsis F-box-Nictaba gene is a stress-inducible gene responsive to salicylic acid, bacterial infection and heat stress, and is involved in salicylic acid related plant defense responses [25]. In rice (Oryza sativa) F-box gene MEIOTIC F-BOX (MOF), which is essential for male meiotic progression was studied, mof meiocytes display disrupted telomere bouquet formation, impaired pairing and synapsis of homologous chromosomes, and arrested meiocytes at late prophase I, followed by apoptosis [26]. DEAD-box proteins are ubiquitous in RNAmediated processes and function by coupling cycles of ATP binding and hydrolysis to changes in affinity for single-stranded RNA. AtRH7, one of the Arabidopsis thaliana DEAD-box RNA helicases is an interactor of Arabidopsis COLD SHOCK DOMAIN PROTEIN 3 (AtCSP3), which is an RNA chaperone involved in cold adaptation [27]. Many disease resistance (R) proteins in plants detect the presence of disease-causing bacteria, viruses, or fungi by recognizing specific pathogen effector molecules that are produced during the infection process [28]. NBS-LRR disease resistant gene are isolated and characterized from Pea (Pisum sativum) [29], Mango [30] which shows that NBS genes recognize many different pathogenic virulence factors and play a very important role in disease defence.

Gene annotations of miRNA targets showed they belong to all three GO categories; molecular function, cellular component and biological process. Molecular functions were highlighted with metabolism, transcription, signal transduction, development and response to stress. Cellular component was enriched with nucleus and biological process was enriched with binding and enzyme activity as depicted in Figure 2 and the GO terms for identified targets are given in Suppl. 3. 
Suppl. 2: Targets genes identified for conserved miRNAs in Niger.

\begin{tabular}{|c|c|c|}
\hline miRNA_Acc. & Target_Acc. & Target Description \\
\hline \multirow{4}{*}{ Gab-miR1 } & Brara.B00171.1 & HMG-box (high mobility group) DNA-binding family protein \\
\hline & Brara.I04066.1 & F-box/RNI-like superfamily protein \\
\hline & Brara.C00853.1 & F-box/RNI-like superfamily protein \\
\hline & Brara.D00129.1 & cytochrome P450, family 76 , subfamily C, polypeptide 4 \\
\hline \multirow{45}{*}{ Gab-miR2 } & Brara.A02187.1 & Pentatricopeptide repeat (PPR) superfamily protein \\
\hline & Brara.G02611.1 & P-loop containing nucleoside triphosphate hydrolases superfamily protein \\
\hline & Brara.C03329.1 & 3-phosphoinositide-dependent protein kinase-1, putative \\
\hline & Brara.D02484.1 & Rhodanese/Cell cycle control phosphatase superfamily protein \\
\hline & Brara.F02877.1 & Frigida-like protein \\
\hline & Brara.I01711.1 & NAD-dependent epimerase/dehydratase family protein \\
\hline & Brara.J02674.1 & Chalcone-flavanone isomerase family protein \\
\hline & Brara.E02713.1 & Tetratricopeptide repeat (TPR)-like superfamily protein \\
\hline & Brara.E00714.6 & acetyl Co-enzyme a carboxylase carboxyltransferase alpha subunit \\
\hline & Brara.E00714.5 & acetyl Co-enzyme a carboxylase carboxyltransferase alpha subunit \\
\hline & Brara.E00714.4 & acetyl Co-enzyme a carboxylase carboxyltransferase alpha subunit \\
\hline & Brara.E00714.3 & acetyl Co-enzyme a carboxylase carboxyltransferase alpha subunit \\
\hline & Brara.C04527.1 & alpha/beta-Hydrolases superfamily protein \\
\hline & Brara.E00714.2 & acetyl Co-enzyme a carboxylase carboxyltransferase alpha subunit \\
\hline & Brara.E00714.1 & acetyl Co-enzyme a carboxylase carboxyltransferase alpha subunit \\
\hline & Brara.B03601.1 & DEAD box RNA helicase (RH3) \\
\hline & Brara.F02796.1 & DEAD box RNA helicase (RH3) \\
\hline & Brara.A00236.1 & Disease resistance protein (TIR-NBS-LRR class) family \\
\hline & Brara.H01668.1 & Disease resistance protein (TIR-NBS-LRR class) family \\
\hline & Brara.D00663.1 & Pentatricopeptide repeat (PPR) superfamily protein \\
\hline & Brara.H02781.1 & basic helix-loop-helix (bHLH) DNA-binding superfamily protein \\
\hline & Brara.E01444.1 & Haloacid dehalogenase-like hydrolase (HAD) superfamily protein \\
\hline & Brara.J01076.1 & Protein kinase superfamily protein \\
\hline & Brara.G03519.2 & Protein of unknown function (DUF630 and DUF632) \\
\hline & Brara.G03519.1 & Protein of unknown function (DUF630 and DUF632) \\
\hline & Brara.A01418.1 & growth-regulating factor 8 \\
\hline & Brara.D02744.1 & growth-regulating factor 9 \\
\hline & Brara.I01630.1 & cytochrome P450, family 96, subfamily A, polypeptide 15 \\
\hline & Brara.D00563.1 & growth-regulating factor 4 \\
\hline & Brara.B01438.1 & growth-regulating factor 7 \\
\hline & Brara.G01562.1 & growth-regulating factor 4 \\
\hline & Brara.C01828.1 & growth-regulating factor 4 \\
\hline & Brara.I03590.1 & growth-regulating factor 4 \\
\hline & Brara.D02218.1 & growth-regulating factor 3 \\
\hline & Brara.E00841.1 & growth-regulating factor 3 \\
\hline & Brara.J00997.1 & Leucine-rich repeat (LRR) family protein \\
\hline & Brara.K00742.1 & growth-regulating factor 8 \\
\hline & Brara.K01237.1 & growth-regulating factor 2 \\
\hline & Brara.A00122.1 & growth-regulating factor 2 \\
\hline & Brara.C02492.1 & growth-regulating factor 1 \\
\hline & Brara.D01740.1 & GRAS family transcription factor \\
\hline & Brara.A00536.1 & $2 \mathrm{Fe}-2 \mathrm{~S}$ ferredoxin-like superfamily protein \\
\hline & Brara.C01276.1 & HEAT SHOCK PROTEIN 81.4 \\
\hline & Brara.J02094.1 & Aldolase-type TIM barrel family protein \\
\hline & Brara.B00492.1 & Aldolase-type TIM barrel family protein \\
\hline
\end{tabular}

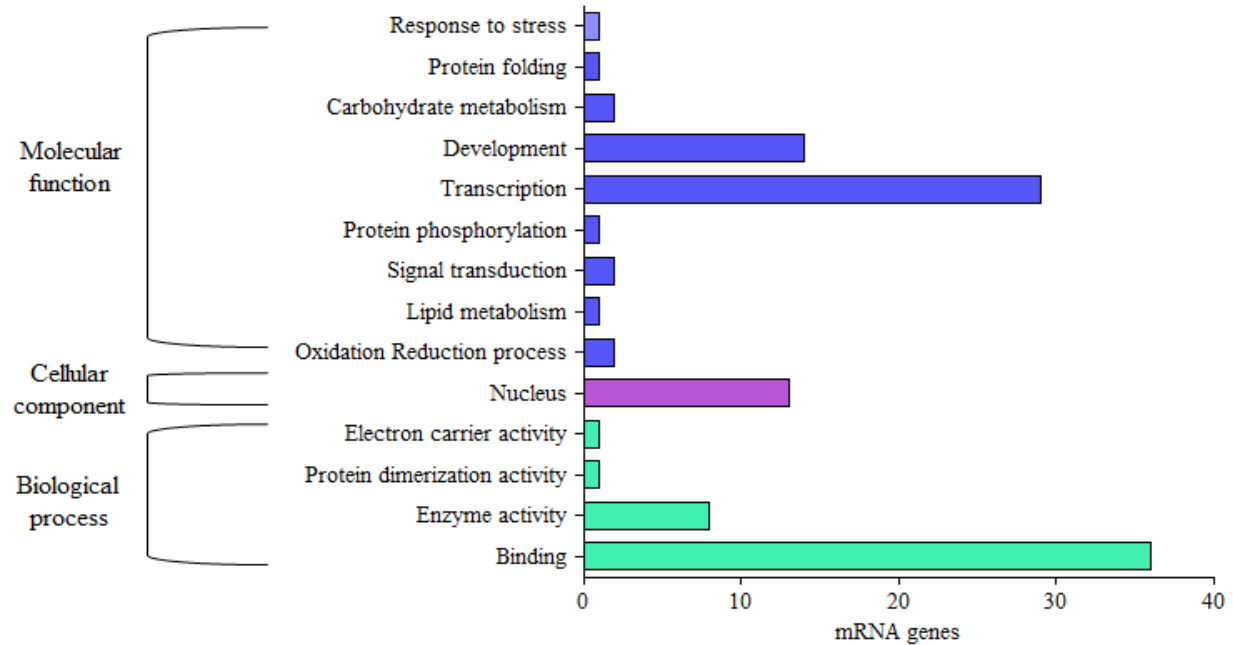

Fig. 2: Gene ontology classification of predicted targets genes for miRNAs identified in Niger. 
Suppl. 3: Gene ontology annotations of miRNAs in Niger.

\begin{tabular}{|c|c|c|}
\hline miRNA_Acc. & Target_Acc. & GO term \\
\hline \multirow{3}{*}{ Gab-miR1 } & Brara.B00171.1 & GO:0005515 \\
\hline & Brara.I04066.1 & GO:0005515 \\
\hline & Brara.C00853.1 & GO:0005506, GO:0016705, GO:0020037, GO:0055114 \\
\hline \multirow{29}{*}{ Gab-miR2 } & Brara.J02674.1 & GO:0016872 \\
\hline & Brara.C04527.1 & GO:0006629 \\
\hline & Brara.B03601.1 & GO:0003723, GO:0004386, GO:0005634, GO:0003677, GO:0005524, GO:0016787, GO:0003676, GO:0008270 \\
\hline & Brara.F02796.1 & GO:0003723, GO:0004386, GO:0005634, GO:0003677, GO:0005524, GO:0016787, GO:0003676, GO:0008270 \\
\hline & Brara.A00236.1 & GO:0043531, GO:0005515, GO:0007165 \\
\hline & Brara.H01668.1 & GO:0043531, GO:0005515, GO:0007165 \\
\hline & Brara.H02781.1 & GO:0046983 \\
\hline & Brara.J01076.1 & GO:0004672, GO:0006468 \\
\hline & Brara.G03519.2 & GO:0006351, GO:0032502, GO:0005524, GO:0005634, GO:0006355 \\
\hline & Brara.G03519.1 & GO:0006351, GO:0032502, GO:0005524, GO:0005634, GO:0006355 \\
\hline & Brara.A01418.1 & GO:0006351, GO:0032502, GO:0005524, GO:0005634, GO:0006355 \\
\hline & Brara.D02744.1 & GO:0006351, GO:0032502, GO:0005524, GO:0005634, GO:0006355 \\
\hline & Brara.I01630.1 & GO:0005506, GO:0016705, GO:0020037, GO:0055114 \\
\hline & Brara.D00563.1 & GO:0005634, GO:0006351, GO:0032502 \\
\hline & Brara.B01438.1 & GO:0006351, GO:0032502, GO:0005524, GO:0005634, GO:0006355 \\
\hline & Brara.G01562.1 & GO:0006351, GO:0032502, GO:0005524, GO:0005634, GO:0006355 \\
\hline & Brara.C01828.1 & GO:0006351, GO:0032502, GO:0005524, GO:0005634, GO:0006355 \\
\hline & Brara.I03590.1 & GO:0006351, GO:0032502, GO:0005524, GO:0005634, GO:0006355 \\
\hline & Brara.D02218.1 & GO:0006351, GO:0032502, GO:0005524, GO:0005634, GO:0006355 \\
\hline & Brara.E00841.1 & GO:0006351, GO:0032502, GO:0005524, GO:0005634, GO:0006355 \\
\hline & Brara.J00997.1 & GO:0005515 \\
\hline & Brara.K00742.1 & GO:0006351, GO:0032502, GO:0005524, GO:0005634, GO:0006355 \\
\hline & Brara.K01237.1 & GO:0006351, GO:0032502, GO:0005524, GO:0005634, GO:0006355 \\
\hline & Brara.A00122.1 & GO:0006351, GO:0032502, GO:0005524, GO:0005634, GO:0006355 \\
\hline & Brara.C02492.1 & GO:0006351, GO:0032502, GO:0005524, GO:0005634, GO:0006355 \\
\hline & Brara.A00536.1 & GO:0009055, GO:0051536 \\
\hline & Brara.C01276.1 & GO:0005524, GO:0006457, GO:0006950, GO:0051082 \\
\hline & Brara.J02094.1 & GO:0005975 \\
\hline & Brara.B00492.1 & GO:0005975 \\
\hline
\end{tabular}

\subsection{KEGG pathway analysis and Protein interactions}

KEGG pathway analysis showed 07 significantly enriched pathways in Niger such as pentose phosphate pathway, polycyclic aromatic hydrocarbon degradation, Fatty acid biosynthesis and metabolic pathways of glycerolipid, glycerophospholipid, pyruvate and propaanote (Table 2).

Table 2 KEGG analysis for miRNA target genes in Niger.

\begin{tabular}{lll}
\hline KEGG ec & Target_ID & Pathways \\
\hline 2.2 .1 .2 & bra00030 & Pentose phosphate pathway \\
\hline 3.1 .1 .26 & bra00561 & Glycerolipid metabolism \\
\hline \multirow{3}{*}{3.1 .1 .3} & bra00561 & Glycerolipid metabolism \\
& bra00564 & Glycerophospholipid metabolism \\
& bra00030 & Pentose phosphate pathway \\
& bra00624 & Polycyclic aromatic hydrocarbon degradation \\
\hline 3.1 .1 .32 & bra00564 & Glycerophospholipid metabolism \\
\hline \multirow{3}{*}{6.4 .1 .2} & bra00061 & Fatty acid biosynthesis \\
& bra00620 & Pyruvate metabolism \\
& bra00640 & Propanoate metabolism
\end{tabular}

Web based protein-protein interactions of the identified miRNAs showed that putative predicted proteins such as Bra022667, Bra039334, Bra0333281, Bra011781, Bra000575,
Bra006956, and Bra005268 which are growth regulating factors work in network and may play a role in regulation of gene expression (Figure 3).

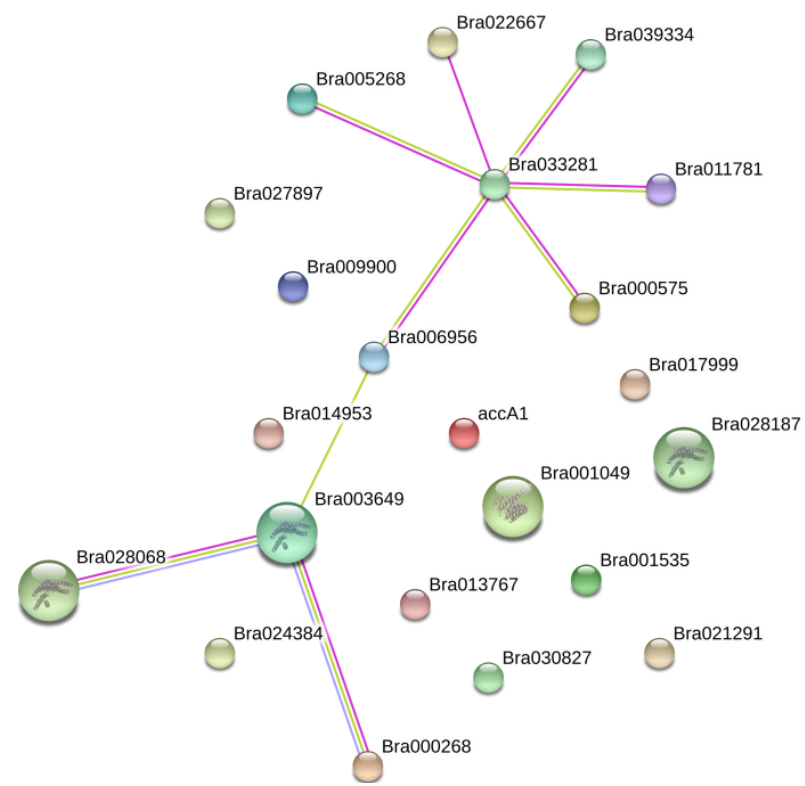

Fig. 3: Protein-protein interaction networks of predicted targets. 


\section{CONCLUSION}

The present study for the first time identifies miRNAs in Niger. The evolutionary conservation of miRNA across the taxa renders powerful approach in their identification using EST analysis. We were able to identify two microRNAs targeting 49 genes. To further analyse the role of miRNAs target interactions, GO analysis and protein interactions of target genes were studied which showed miRNAs may play an important role in growth and development. These finding will contribute for future investigations of miRNAs in Niger under abiotic and biotic stress.

\section{Financial support and sponsorship: Nil.}

Conflict of Interests: There are no conflicts of interest.

\section{REFERENCE}

1. Chen X. Small RNAs and their roles in plant development. Annual review of cell and developmental biology 2009; 25:21-44.

2. Li YF, Zheng Y, Addo-Quaye C, Zhang L, Saini A, Jagadeeswaran G, et al. Transcriptome-wide identification of microRNA targets in rice. The Plant journal: for cell and molecular biology 2010; 62(5):742-59.

3. Martinez G, Forment J, Llave C, Pallas V, Gomez G. High-throughput sequencing, characterization and detection of new and conserved cucumber miRNAs. PloS one 2011; 6(5):e19523.

4. Carrington JC, Ambros V. Role of micro RNAs in plant and animal development. Science 2003; 301:336-338.

5. Sunkar R, Li YF, Jagadeeswaran G. Functions of micro RNAs in plant stress responses. Trends in Plant Science 2012; 17(4):196-203.

6. Zhang B, Pan X, Anderson TA. Identification of 188 conserved maize microRNAs and their targets. FEBS letters 2006; 580(15):3753-62.

7. Allen E, Xie Z, Gustafson AM, Sung GH, Spatafora JW, Carrington JC. Evolution of microRNA genes by inverted duplication of target gene sequences in Arabidopsis thaliana. Nature genetics 2004; 36(12):1282-90

8. Fahlgren N, Howell MD, Kasschau KD, Chapman EJ, Sullivan CM, Cumbie JS, et al. High-throughput sequencing of Arabidopsis microRNAs: evidence for frequent birth and death of MIRNA genes. PloS one 2007; 2(2):e219.

9. Yong Huang, Quan Zou, Shun Ming Tang, Li Gang Wang, Xing JiaShen. Computational identification and characteristics of novel microRNAs from the silkworm (Bombyx mori L.). Molecular Biology Reports 2010; 37(7): 3171-3176.

10. Xi Hong, Ling Ping Zhao, Quan Zou, Zhan Bin Wang. Identification of MicroRNA Genes and their mRNA Targets in Festuca arundinacea. Applied Biochemistry and Biotechnology 2014; 172(8): 3875-3887.

11. Huang Y, Zou Q, Wang ZB. Computational identification of miRNA genes and their targets in mulberry. Russian Journal of Plant Physiology 2014; 61(4): 537-542.

12. LIU Yong-xin, CHANG Wei, HAN Ying-peng, ZOU Quan, GUO Mao-zu3 and LI Wen-bin. In silico Detection of Novel MicroRNAs Genes in Soybean Genome. Agricultural Sciences in China 2011; 10(9): 1336-1345

13. Usha S, Jyothi MN, Suchithra B, Rekha Dixit, Rai DV, Nagesh Babu R. Computational identification of micro RNAs and their targets from Finger millet (Eleusine coracana). Interdisciplinary Sciences Computational Life Sciences. 2015; doi: 10.1007/s12539-015-0130-y.

14. Huang X, Madan A. CAP3: A DNA sequence assembly program. Genome Res. 1999; 9:868-877

15. Zuker M. Mfold web server for nucleic acid folding and hybridization prediction. Nucleic Acids Res. 2003; 31:3406-3415.
16. Leyi Wei, Minghong Liao, Yue Gao, Rongrong Ji, Zengyou He, Quan Zou. Improved and Promising Identification of Human MicroRNAs by Incorporating a High-quality Negative Set. IEEE/ACM Transactions on Computational Biology and Bioinformatics 2013; [Epub ahead of print].

17. Xinbin Dai, Patrick $X$ Zhao. psRNATarget: A Plant Small RNA Target Analysis Server. Nuc. Aci. Res. 2011; 39:W155-W159.

18. Ye J, Fang L, et al. Nucleic Acids Res. 2006; 34(Web service issue), 293-297.

19. Ambros V, Bartel B, Bartel DP. A uniform system for microRNA annotation. RNA 2003; 9:277-279.

20. Chi X, Yang Q, Chen $X$, Wang $J$ et al. Identification and characterization of microRNAs from peanut (Arachis hypogaea L.) by high throughput sequencing. PLoS One 2011; 1 6: e27530.

21. Xu L, Wang Y, Xu Y, Wang L, Zhai L et al. Identification and characterization of novel and conserved microRNAs in radish (Raphanus sativus L.) using high-throughput sequencing. Plant Science 2013; 201-202: 108-114.

22. Han Y, Luan F, Zhu H, Shao Y, Chen A, Lu C, Luo Y, Zhu B. Computational identification of microRNAs and their targets in Wheat (Triticum aestivum L.). Science in China Series C-Life Sciences 2009; 52:1091-1100.

23. Ikeda Y, Kinoshita Y, Susaki D, Ikeda Y, Iwano M, Takayama S, Higashiyama T, Kakutani T, Kinoshita T. HMG domain containing SSRP1 is required for DNA demethylation and genomic imprinting in Arabidopsis. Dev Cell. 2011; 21(3):589-96.

24. Roy A, Dutta A, Roy D, Ganguly P, Ghosh R, Kar RK, Bhunia A, Mukhobadhyay J, Chaudhuri S. Deciphering the role of the AT-rich interaction domain and the HMG-box domain of ARID-HMG proteins of Arabidopsis thaliana. Plant Mol Biol. 2016; 92(3):371-88.

25. Stefanowicz K, Lannoo N, Zhao Y, Eggermont L, Van Hove J, Al Atalah B, Van Damme EJ. Glycan-binding F-box protein from Arabidopsis thaliana protects plants from Pseudomonas syringae infection. BMC Plant Biol. 2016; 16(1):213.

26. He Y, Wang C, Higgins JD, Yu J, Zong J, Lu P, Zhang D, Liang W. MEIOTIC F-BOX Is Essential for Male Meiotic DNA Double-Strand Break Repair in Rice. Plant Cell 2016; 28(8):1879-93.

27. Liu Y, Tabata D, Imai R. A Cold-Inducible DEAD-Box RNA Helicase from Arabidopsis thaliana Regulates Plant Growth and Development under Low Temperature. PLoS One 2016; 11(4):e0154040.

28. Martin GB, Bogdanove AJ, Sessa G. Understanding the functions of plant disease resistance proteins. Annu Rev Plant Biol. 2003; 54:2361

29. Djebbi S, Bouktila D, Makni H, Makni M, Mezghani-Khemakhem M. Identification and characterization of novel NBS-LRR resistance gene analogues from the pea. Genet Mol Res. 2015; 14(2):6419-28.

30. Lei X, Yao Q, Xu X, Liu Y. Isolation and characterization of NBSLRR resistance gene analogues from mango. Biotechnol Biotechnol Equip. 2014; 28(3):417-424.

How to cite this article:

Prathiba KY, Usha S, Suchithra B, Jyothi MN, Devaraj VR, Nagesh babu R. Computational identification of miRNAs and their targets from Niger (Guizotia abyssinica). J App Biol Biotech. 2017; 5 (02): 053-058. 